\title{
SIMPLIFICAÇÃO OPERACIONAL EM LEVANTAMENTOS GEODÉSICOS COM O EMPREGO DE INSTRUMENTOS ELETRONNICOS
}

\author{
Moacyr de CARVALHO *
}

\section{RESUMO}

\begin{abstract}
Este trabalho trata de certas simplificações operacionais em levantamentos geodésicos para fins de projetos ou cartografia, mediante o correlacionamento linear entre duas variáveis quantitativas.

Tais variáveis são as constituídas por coeficientes de refração, curvatura e índice de refração extraídas de amostra observada em 80 pares dessas variáveis.

O trabalho contém uma análise conclusiva estabelecendo condiçőes limites para a sua aplicação.
\end{abstract}

\begin{abstract}
This work deals with some operational simplifications about geodetical surveys with the aim of cartographical planning, by means of the establishment of linear correlation between quantitative variables.

Such variables are constituted by curvature refraction coefficient and refraction index calculated from a sample with a eighty variables.

The work contains a conclusive analysis which gives limited conditions to its application.
\end{abstract}

\section{INTRODUÇĀO}

O presente trabalho gira em torno do tratamento estatístico de 80 pares de dados de observação de coeficientes de refração e curvatura, notado Ccr, e dos índices de refração $\mathrm{n}$, obtidos respectivamente para o controle de alturas zenitais de visadas recíprocas e para a correção de medidas de distâncias em poligonais eletrônicas, obtidas com telurômetros modelos MRA.1 e MRA.3.

Os dados em questão resultaram do desenvolvimento de poligonais para fins de locação plano-altimétrica de diferentes poços perfurados para coleta de dados e testes, durante a pesquisa de águas subterrâneas na bacia do rio São José dos Dourados e do médio Tietê.

A determinação, com precisão coerente, da locação de poços foi considerada necessária para o estudo da geometria de aquíferos, bem como a determinação topográfica das altitudes de boca, posição do lençol freático e profundidades atingidas, com amarração a um sistema de referência geodésica uniforme e confiável.

Esta referência é a constituída pelo sistema oficial da rede de triangulação de $1 .^{\mathrm{a}}$ ordem do Estado de São Paulo, bem como de sua rede de nivelamento de precisão.

Também, entre os dados, estão incluídos aqueles obtidos no projeto de mapeamento do Estado, na escala de $1 / 50.000$, desenvolvido na mesma região.

\section{OBJETIVO}

Foi o de comprovar a hipótese de correlação estatística entre os dados experimentais (observações) de $\mathrm{Ccr}$ e $\mathrm{n}$, respectivamente usados para o controle operacional de distâncias zenitais e obtenção da distância geodésica entre estações de poligonais com a utilização de equipamentos eletrônicos.

\footnotetext{
* Instituto Geológico - Caixa Postal 8772 - 01000 - São Paulo, SP, Brasil
} 
A comprovação de tal hipótese teria a conseqüência de simplificar as operações de campo pela redução de deslocamentos da equipe de campo e das observações e, por conseguinte, do tempo operacional, pela razão de que os vértices consecutivos em poligonais eletrônicas usualmente estão separados por distâncias entre 15 a $30 \mathrm{~km}$ ou mais.

A determinação, no campo, de Ccr, é feita pela fórmula seguinte:

$$
\mathrm{Ccr}=\frac{\left(\mathrm{Z}_{2}+\mathrm{Z}_{1}-180^{\circ}\right) \cdot 0,145}{\mathrm{~d}}
$$

a qual é comparada com o valor 0,067, critério da aceitação ou rejeição das observações.

$$
\begin{aligned}
\mathrm{Z}_{1}= & \text { distância zenital no ponto } 1 \\
\mathrm{Z}_{2}= & \text { distância zenital no ponto } 2 \\
\mathrm{~d}= & \text { distância fornecida pelo } \\
& \text { telurômetro }
\end{aligned}
$$

Pela equação de regressão obtida neste trabalho como conclusão principal, é possível a determinação de $\mathrm{n}^{\prime}=\frac{\mathrm{n}_{0}}{\mathrm{n}}$ aue possibilita o cálculo das distâncias geodésicas, com a eliminação de tabelas e gráficos diversos usualmente enpregados no esquema de cálculo.

$\mathrm{n}_{0}=1,000325 \quad \mathrm{n}=$ valor obtido pela equação de regressão

\section{A colocação do problema}

O índice de refração, elemento básico para o cálculo da distância geodésica entre dois pontos, na rotina de cálculo do sistema UTM (Universe Transverse Mercator), hoje largamente empregado em cartografia e em levantamentos geodésicos de apoio, é obtido normalmente pela fórmula de Essen-Froome seguinte:

$$
\begin{aligned}
& (n-1) \cdot 10^{6}=\frac{4730}{459.688+t}(P+E) \\
& \text { Onde } E=\frac{8540}{459.698} e^{\prime} \\
& \text { e } e=e^{\prime}-0,000367 p\left(t-t^{\prime}\right) \\
& \left(1+\frac{t^{\prime}-32}{1571}\right)
\end{aligned}
$$

sendo

$\mathrm{n}=$ índice de refração

$\mathrm{e}=$ tensão parcial do vapor dágua $\mathrm{pol} / \mathrm{Hg}$

$\mathrm{e}^{\prime}=$ tensão máxima de vapor

$\mathrm{t}=$ temperatura do termômetro seco $\mathrm{F}^{\circ}$

$t^{\prime}=$ temperatura do termômetro úmido $\mathrm{F}^{\circ}$

$\mathrm{p}=$ pressão barométrica em $\mathrm{pol} / \mathrm{Hg}$

Assim, a fórmula acima leva em conta basicamente as condições termodinâmicas da atmosfera, no momento em que se efetuam as medidas do tempo de percurso de ondas eletromagnéticas (faixas de microondas), resultando determinações de $\mathrm{n}$ mais coerentes com a realidade.

Não obstante, os parâmetros de estado do ar variam continuamente em cada tempo, como função do tempo, na trajetória das ondas eletromagnéticas enquanto que, no dado momento das operações com o telurômetro, se tomam medidas de temperatura e pressão somente em dois pontos (nas estações), o que inevitavelmente conduz a uma certa imprecisão na determinação de $n$.

Experiências elucidativas foram conduzidas na Suíça, em medições de distâncias com equipamentos eletrônicos, em direções transversais simultaneamente, com resultados que indicam a influência da variabilidade citada. Ademais se sabe que existem variáveis aleatórias não identificadas, afetando o índice de refração, como outras fontes de imprecisão, sem todavia tornar, inconveniente na prática, o uso da fórmula de Essen-Froome.

No que se refere aos dados Ccr, assinalamos que na prática da poligonação eletrônica eles servem como elemento de controle de medidas zenitais, tomando-se para efeito de comparação o valor de referência 0,067 . As observações mostram que, apenas em distâncias inferiores a $5 \mathrm{~km}$, os valores calculados de Ccr se afastam inadmissivelmente daquele valor de referência.

A fórmula correntemente utilizada, anteriormente citada, provém de uma concepção geométrica simples que não leva em conta variações aleatórias que evidentemente influenciam o processo.

Conquanto o índice $\mathrm{n}$ e coeficiente $\mathrm{Ccr}$ apresentem variações ou dispersões em torno de um valor médio, tais dispersões estão contidas num intervalo definido de pequena amplitude, por influência de variáveis aleatórias contínuas. 
Cada uma das variáveis que intervém no processo, atuando independentemente, produzem variações muito pequenas no desvio total, cumprindo assim as condições do teorema de Liapunov, podendo-se pois supor que os erros se distribuem segundo leis normais.

Como consequiência destes raciocínios foi levantada a hipótese da correlação entre dados experimentais de valores de $\mathrm{n}$ e $\mathrm{Ccr}$, e assim testar o grau de correlação entre os 80 pares de valores coletados de $\mathrm{n}$ e Ccr, com a introdução da idéia de uma regressão da forma linear.

O tratamento estatístico, análises e conclusões ou resultados são os que seguem adiante.

$\mathrm{x}=\mathrm{Ccr}$ (coeficiente de refração e curvatura)

\begin{tabular}{|c|c|c|c|}
\hline $\mathrm{x}$ & $f_{x}$ & $\mathrm{x}_{\mathrm{m}}$ & $\mathrm{f}_{\mathrm{x}} \mathrm{x}_{\mathrm{m}}$ \\
\hline $06047-6139$ & 3 & 0,06093 & 0,182 \\
\hline $06139-6231$ & 0 & 0,06185 & 0 \\
\hline ,06231-6323 & 3 & 0,06277 & 0,188 \\
\hline $0,06323-6415$ & 0 & 0,06369 & 0 \\
\hline $0,06415-6507$ & 3 & 0,06461 & 0,193 \\
\hline 0,06507-6599 & 5 & 0,06553 & 0 , \\
\hline $0,06599-6691$ & 3 & 0,06645 & 0,19 \\
\hline $0,06691-6783$ & 4 & 0,06737 & 0,269 \\
\hline $0,06783-6875$ & 6 & 0,06828 & 0,4097 \\
\hline $0,06875-6967$ & 8 & 0,06921 & 0,5536 \\
\hline $0,06967-7059$ & 13 & 0,07013 & 0,9116 \\
\hline 0,07059-7151 & 6 & 0,07105 & 0,4263 \\
\hline $0,07151-7243$ & 7 & 0,07197 & 0,5037 \\
\hline 0,07243-7335 & 4 & 0,072 & 0,291 \\
\hline $5-7427$ & 7 & 0,073 & 0,5166 \\
\hline 7-7519 & 3 & 0,07 & 0,224 \\
\hline 9-7611 & 2 & 0,07 & 0,1513 \\
\hline $1-7703$ & 1 & 0,07 & 0,0765 \\
\hline 0,07 & 0 & 0,07 & 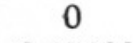 \\
\hline 0,07795-7887 & 2 & 0,07841 & 0,1568 \\
\hline \multicolumn{3}{|c|}{$\begin{array}{l}\Sigma 80 \\
=0,06979\end{array}$} & $J$, \\
\hline
\end{tabular}

$\mathrm{O}$ valor de referência utilizado para controle de medidas de distâncias zenitais indicado atrás foi de 0,067 . Na distribuição acima verfica-se que a maior freqüência registrada corresponde ao intervalo de valores observados na classe 0,06967-7059, com frequiência 13 , sendo que o valor médio é de 0,07013 . A média aritmética é 0,06979 , praticamente 0,070 .
Isto sugere que o valor de referência a ser adotado deveria ser de 0,070 ao invés do valor 0,067 .

A fórmula que dá a diferença de nível entre dois pontos $\mathrm{A}$ e $\mathrm{B}$ é:

$h_{B}-h_{A}=d \operatorname{tg} a+C c r \cdot d^{2}+i-s$

onde:

$$
\begin{aligned}
& \mathrm{a}=\text { ângulo de inclinação } \\
& \mathrm{d}=\text { distância em km } \\
& \mathrm{i}=\text { altura do instrumento } \\
& \mathrm{s}=\text { altura do sinal visado }
\end{aligned}
$$

Façamos a $=0$ e i $=\mathrm{s}$ em (1). Vem

$\Delta \mathrm{h}=\mathrm{h}_{\mathrm{B}}-\mathrm{h}_{\mathrm{A}}=\mathrm{Ccr} \cdot \mathrm{d}^{2}$ diferença devido à refração e curvatura.

Consideremos $\Delta h=f(C c r)$, isto é, Ccr variável e derivemos esta função em relação a Ccr.

$$
\mathrm{f}^{\prime}(\mathrm{Ccr})=\mathrm{d}^{2} \text { ou } \mathrm{d} \Delta \mathrm{h}=\mathrm{dCcr} \cdot \mathrm{d}^{2}
$$

Tomando-se dCcr pequeno em valor absoluto, isto é, $\Delta \mathrm{h} \simeq \mathrm{dh}$ e apliquemos (2) para o intervalo de variações de Ccr que estamos examinando.

Os afastamentos máximos em relação ao valor de referência sugerido, igual a 0,070, conforme tabela nos dá:

$$
\text { e } \quad \begin{array}{r}
0,070-0,060=0,010 \\
0,078-0,070=0,008
\end{array}
$$

Observemos que as distâncias de lados de poligonais que serviram de base para este estudo variam, geralmente, em torno de 15 km. Para este caso tem-se:

$$
\mathrm{d} \mathrm{h}=0,008 \cdot 15^{2}=1,8 \mathrm{~m}
$$$$
\mathrm{d} h=0,010 \cdot 15^{2}=2,5 \mathrm{~m}
$$

Segundo normas elaboradas pelo grupo de trabalho organizado pelo Instituto PanAmericano de Geografia e História, para a definição de normas de controle geodésico fundamental utilizando instrumentos eletromagnéticos para a medição de distância, ficou estabelecido que, com base em processos do IPGH, o fechamento máximo admissível entre as conexões de nivelamento é de $0,30 \sqrt{\mathrm{d} \mathrm{km}}$.

Em uma distância de $15 \mathrm{~km}$ tem-se $1,16 \mathrm{~m}$ de fechamento máximo admissível, valor este compreendido entre 0,075 e 0,065 , que compreendem 68 dos 80 casos estudados.

Em resumo, vê-se que, em poligonais com utilização de instrumentos eletromagnéticos, para controle geodésico fundamental o 


\section{CÁLCULO DOS PARÂMETROS DA DISTRIBUIÇÃO DOS COEFICIENTES DE REFRAÇÃO E CURVATURA}

\begin{tabular}{|c|c|}
\hline$f_{x_{i}}$ & $\left(x_{i}-x_{0}\right) \cdot 10^{-5}$ \\
\hline 3 & $(6093-6979) \cdot 10^{-5}$ \\
\hline 0 & $(6185-6979) \cdot 10^{-5}$ \\
\hline 3 & $(6277-6979) \cdot 10^{-5}$ \\
\hline 0 & $(6369-6979) \cdot 10^{-5}$ \\
\hline 3 & $(6461-6979) \cdot 10^{-5}$ \\
\hline 5 & $(6553-6979) \cdot 10^{-5}$ \\
\hline 3 & $(6645-6979) \cdot 10^{-5}$ \\
\hline 4 & $(6737-6979) \cdot 10^{-5}$ \\
\hline 6 & $(6828-6979) \cdot 10^{-5}$ \\
\hline 8 & $(6921-6979) \cdot 10^{-5}$ \\
\hline 13 & $(7013-6979) \cdot 10^{-5}$ \\
\hline 6 & $(7105-6979) \cdot 10^{-5}$ \\
\hline 7 & $(7197-6979) \cdot 10^{-5}$ \\
\hline 4 & $(7289-6979) \cdot 10^{-5}$ \\
\hline 7 & $(7381-6979) \cdot 10^{-5}$ \\
\hline 3 & $(7473-6979) \cdot 10^{-5}$ \\
\hline 2 & $(7565-6979) \cdot 10^{-5}$ \\
\hline 1 & $(7749-6979) \cdot 10^{-5}$ \\
\hline 0 & $(7841-6979) \cdot 10^{-5}$ \\
\hline 2 & $(7841-6979) \cdot 10^{-5}$ \\
\hline
\end{tabular}

$\left(x_{i}-x_{0}\right)^{2} \cdot f_{x_{1}}\left(10^{-5}\right)^{2}$

$2654988 \cdot 10^{-10}$

0

$1478412 \cdot 10^{-10}$

0

$804972 \cdot 10^{-10}$

$994580 \cdot 10^{-10}$

$334668 \cdot 10^{-10}$

$234256 \cdot 10^{-10}$

$136806 \cdot 10^{-10}$

$26912 \cdot 10^{-10}$

$15028 \cdot 10^{-10}$

$95256 \cdot 10^{-10}$

$332668 \cdot 10^{-10}$

$384400 \cdot 10^{-10}$

$1131228 \cdot 10^{-10}$

$732108 \cdot 10^{-10}$

$686792.10^{-10}$

$459684 \cdot 10^{-10}$

0

$1486088 \cdot 10^{-10}$

$\Sigma 11601635 \cdot 10^{-10}$

$$
\begin{aligned}
& \mathrm{n}=80 \\
& \mathrm{x}_{0}=0,06979
\end{aligned}
$$

$$
s=10^{-5} x \sqrt{\frac{\Sigma\left(x_{i}-X_{0}\right)^{2} \cdot f_{x i}}{n-1}=0,00383}
$$

$\mathrm{s}=$ desvio quadrático médio corrigido

termo $\mathrm{Ccr} . \mathrm{d}^{2}$ da fórmula (1) deve ser levada em conta, quando não se usa o método de visadas recíprocas e simultâneas, mas pode ser desprezado no caso de pontos isolados, quando a distância não supere $15 \mathrm{~km}$.

Porém, inversamente ao que se propôs a análise deste trabalho, poder-se-ia examinar em que condições se pode determinar Ccr a partir de $\mathrm{n}$ calculado, mediante a hipótese de regressão linear, tal que um nivelamento trigonométrico em poligonais a telurômetro possa prescindir das visadas recíprocas, substituindo-as por apenas uma medida do ângulo de inclinação. 
Polígono de frequência e curvo normal teórica de parâmetros $\quad X m=0,06979$ $s=0,00225$

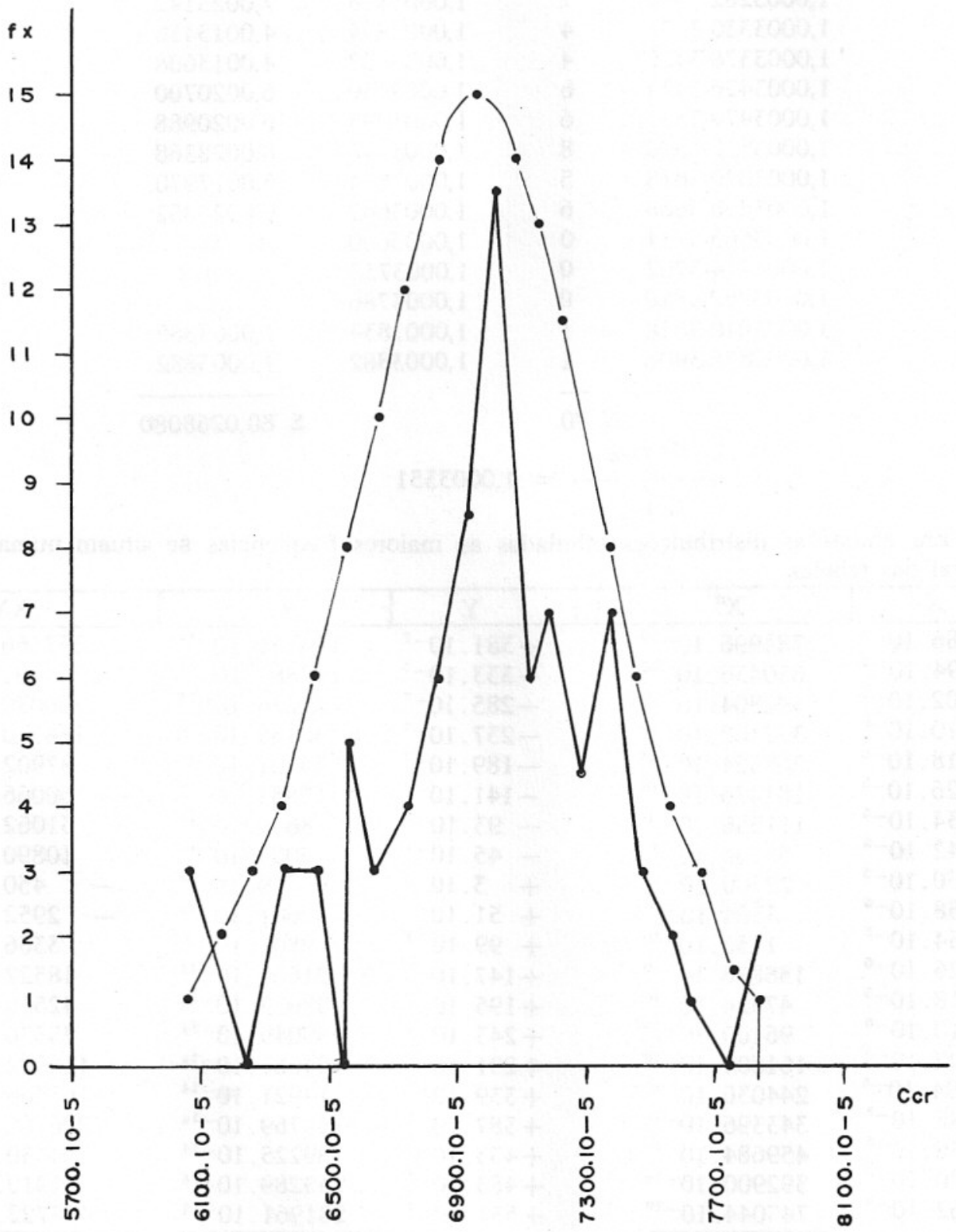

Figura 1 
Rev. IG, São Paulo, 4(1/2):53-65, jan./dez. 1983

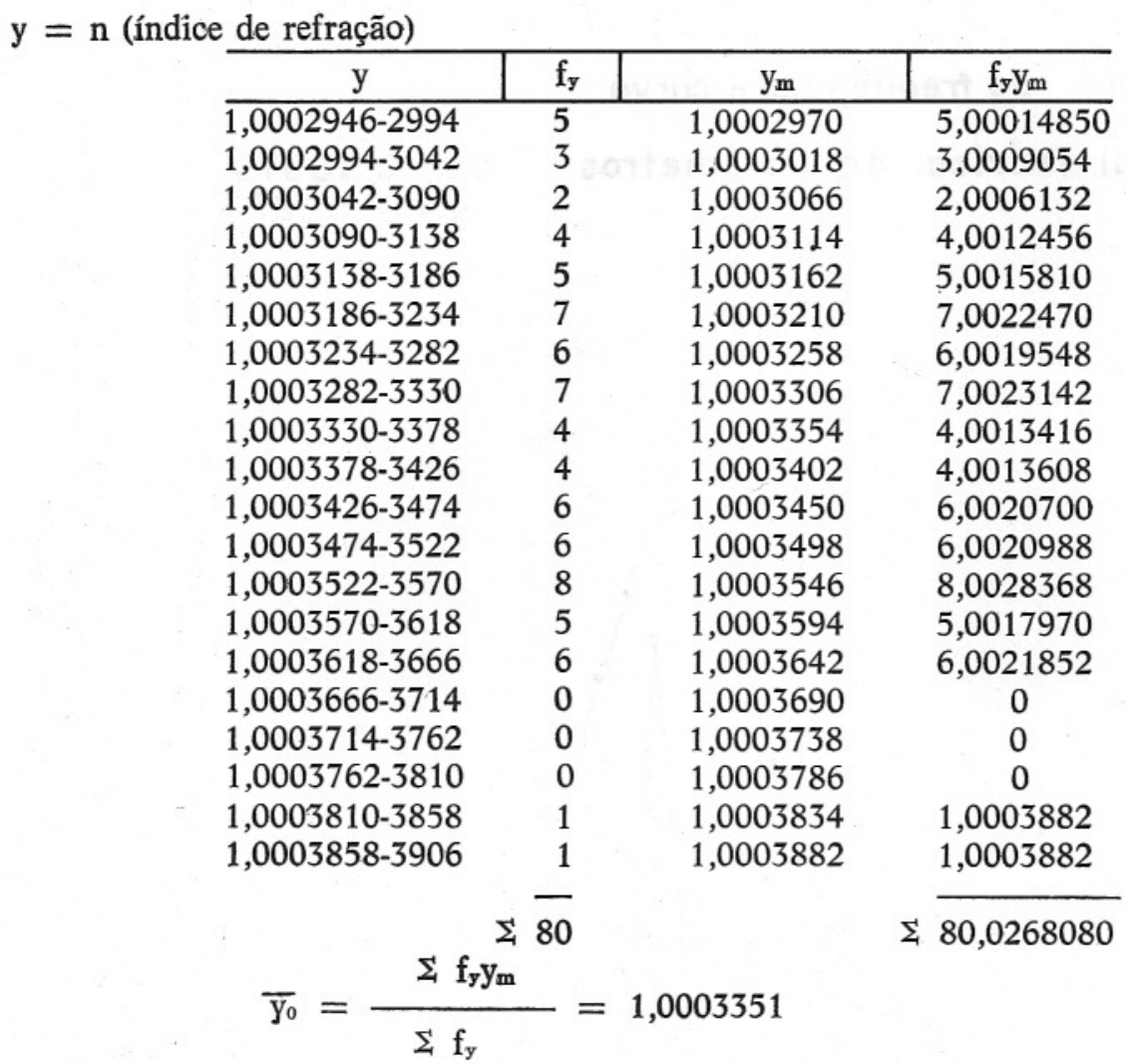

Em ambas as distribuições tabuladas as maiores freqüências se situam numa faixa central das tabelas.

\begin{tabular}{|c|c|c|c|c|}
\hline $\bar{X}$ & $X^{2}$ & Y & $\mathrm{Y}^{2}$ & $\mathrm{XY}$ \\
\hline$-886.10^{-5}$ & $784996.10^{-10}$ & $-381.10^{-7}$ & $145161.10^{-14}$ & $337566.10^{-12}$ \\
\hline$-794.10^{-5}$ & $630436 \cdot 10^{-10}$ & $-333.10^{-7}$ & $110889.10^{-14}$ & $264402.10^{-12}$ \\
\hline$-702.10^{-5}$ & $492804 \cdot 10^{-10}$ & $-285 \cdot 10^{-7}$ & $81225.10^{-14}$ & $200070.10^{-12}$ \\
\hline$-610.10^{-5}$ & $372100 \cdot 10^{-10}$ & $-237.10^{-7}$ & $56169.10^{-14}$ & $144570.10^{-12}$ \\
\hline$-518 \cdot 10^{-5}$ & $268324 \cdot 10^{-10}$ & $-189.10^{-7}$ & $35721.10^{-14}$ & $97902.10^{-12}$ \\
\hline$-426.10^{-5}$ & $181476.10^{-10}$ & $-141.10^{-7}$ & $19881.10^{-14}$ & $60066 \cdot 10^{-12}$ \\
\hline$-334.10^{-5}$ & $111556.10^{-10}$ & $-93.10^{-7}$ & $8649 \cdot 10^{-14}$ & $31062 \cdot 10^{-12}$ \\
\hline$-242.10^{-5}$ & $58564.10^{-10}$ & $-45.10^{-7}$ & $2025.10^{-14}$ & $10890.10^{-12}$ \\
\hline$-150.10^{-5}$ & $22500 \cdot 10^{-10}$ & $+\quad 3.10^{-7}$ & $9.10^{-14}$ & $450.10^{-12}$ \\
\hline$-58.10^{-5}$ & $3364: 10^{-10}$ & $+51.10^{-7}$ & $2601.10^{-14}$ & $2952.10^{-12}$ \\
\hline$+34.10^{-5}$ & $1156.10^{-10}$ & $+99.10^{-7}$ & $9801.10^{-14}$ & $3366.10^{-12}$ \\
\hline$+126.10^{-5}$ & $158876.10^{-10}$ & $+147.10^{-7}$ & $21609.10^{-14}$ & $18522.10^{-12}$ \\
\hline$+218.10^{-5}$ & $47524 \cdot 10^{-10}$ & $+195.10^{-7}$ & $38025.10^{-14}$ & $42510 \cdot 10^{-12}$ \\
\hline$+310.10^{-5}$ & $96100 \cdot 10^{-10}$ & $+243.10^{-7}$ & $59049.10^{-14}$ & $75330 \cdot 10^{-12}$ \\
\hline$+402.10^{-5}$ & $161604 \cdot 10^{-10}$ & $+291.10^{-7}$ & $84681 \cdot 10^{-14}$ & $116982 \cdot 10^{-12}$ \\
\hline$+494.10^{-5}$ & $244036 \cdot 10^{-10}$ & $+339.10^{-7}$ & $114921.10^{-14}$ & $167466 \cdot 10^{-12}$ \\
\hline$+586.10^{-5}$ & $343396.10^{-10}$ & $+387.10^{-7}$ & $149769.10^{-14}$ & $226782.10^{-12}$ \\
\hline$+678.10^{-5}$ & $459684 \cdot 10^{-10}$ & $+435.10^{-7}$ & $189225.10^{-14}$ & $294930 \cdot 10^{-12}$ \\
\hline$+770.10^{-5}$ & $592900.10^{-10}$ & $+483.10^{-7}$ & $233289.10^{-14}$ & $371910^{\circ} 10^{-12}$ \\
\hline$+862.10^{-5}$ & $74.3044 .10^{-10}$ & $+531.10^{-7}$ & $281961.10^{-14}$ & $457722.10^{-12}$ \\
\hline & $\Sigma \overline{5631440.10^{-10}}$ & & $\overline{\Sigma 1644660.10^{-14}}$ & $\Sigma 2918640 \cdot 10^{-12}$ \\
\hline
\end{tabular}

hipótese linear 
Equação de regressão

$$
\begin{aligned}
y-y_{0}=\left(\frac{\Sigma X Y}{\Sigma X^{2}}\right)\left(x-x_{0}\right) \\
y-1,0003351=0,0052(x-0,06979) \\
y=0,0052 \times+0,9999722
\end{aligned}
$$

\begin{tabular}{l|c|c}
\hline n coletado & n calculado & desvio \\
\hline & & \\
1,0003286 & 1,0003547 & $+0,0000261$ \\
1,0003511 & 1,0003360 & $-0,0000151$ \\
1,0003546 & 1,0003570 & $+0,0000024$ \\
1,0003333 & 1,0003300 & $-0,0000033$ \\
1,0003441 & 1,0003140 & $-0,0000301$ \\
1,0003534 & 1,0003302 & $-0,0000232$ \\
1,0003141 & 1,0003274 & $+0,0000133$ \\
1,0003300 & 1,0003215 & $-0,0000085$ \\
1,0003288 & 1,0003178 & $-0,0000110$ \\
1,0003263 & 1,0003197 & $-0,0000066$ \\
1,0003303 & 1,0003004 & $-0,0000299$ \\
1,0003281 & 1,0003227 & $-0,0000054$ \\
1,0003110 & 1,0003349 & $+0,0000239$ \\
1,0003327 & 1,0003058 & $-0,0000269$ \\
1,0003017 & 1,0003145 & $+0,0000128$ \\
1,0003223 & 1,0003319 & $+0,0000096$ \\
1,0003170 & 1,0002872 & $+0,0000298$ \\
1,0003114 & 1,0003345 & $+0,0000231$ \\
1,0003114 & 1,0002999 & $-0,0000115$ \\
1,0003482 & 1,0003354 & $-0,0000128$ \\
1,0003451 & 1,0003169 & $-0,0000282$ \\
1,0003357 & 1,0003282 & $-0,0000075$ \\
1,0003190 & 1,0003468 & $+0,0000278$ \\
1,0003273 & 1,0003116 & $-0,0000157$ \\
1,0003217 & 1,0003280 & $+0,0000063$ \\
1,0003473 & 1,0003696 & $+0,0000223$ \\
1,0003400 & 1,0003075 & $-0,0000325$ \\
1,0003615 & 1,0003641 & $+0,0000026$ \\
1,0003632 & 1,0003367 & $-0,0000265$ \\
1,0003288 & 1,0003365 & $+0,0000077$ \\
1,0003506 & 1,0003322 & $-0,0000184$ \\
1,0003620 & 1,0003337 & $+0,0000283$ \\
1,0003185 & 1,0003311 & $+0,0000126$ \\
1,0003212 & 1,0003372 & $+0,0000160$ \\
1,0003280 & 1,0003456 & $+0,0000176$ \\
& & \\
& &
\end{tabular}




\section{DETERMINAÇÃO GRÁFICA DA RETA DE REGRESSÃO}

Para este fim foi usado o conjunto de pontos $\mathrm{M}\left(\mathrm{Ccr}_{i} ; \mathrm{n}_{\mathrm{i}}\right)$ abaixo, em número de 20 .

$\mathrm{O}$ coeficiente da reta $\mathrm{K}=$ tga obtido foi de 0,0054 contra o valor de 0,0052 calculado.

\begin{tabular}{|c|c|c|c|}
\hline \multicolumn{2}{|c|}{$\operatorname{Ccr}_{i}$} & $\left(n_{i}-1\right)$ & . $10^{-7}$ \\
\hline 6093. & $10^{-5}$ & 2970. & $10^{-\tau}$ \\
\hline 6185. & & 3018. & $"$ \\
\hline 6277. & $"$ & 3066. & $"$ \\
\hline 6369. & $"$ & 3114. & $"$ \\
\hline 6461. & $"$ & 3162. & $"$ \\
\hline 6553. & $"$ & 3210. & $"$ \\
\hline 6645. & $"$ & 3258. & $"$ \\
\hline 6737. & $"$ & 3306. & $"$ \\
\hline 6828. & $"$ & 3354. & $"$ \\
\hline 6921. & $"$ & 3402 . & $"$ \\
\hline 7013. & $"$ & 3450. & $"$ \\
\hline 7105 . & $"$ & 3498. & $"$ \\
\hline 7197. & $"$ & 3546. & $"$ \\
\hline 7289. & $"$ & 3594. & $"$ \\
\hline 7381. & ", & 3642. & $"$ \\
\hline 7473 . & 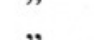 & 3690 . & 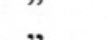 \\
\hline 7565. & 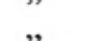 & 3738. & 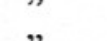 \\
\hline 7657. & $"$ & 3786. & $"$ \\
\hline 7749. & 3 & 3834. & $"$ \\
\hline 7841 . & $10^{-5}$ & 3882 . & $10^{-7}$ \\
\hline
\end{tabular}

O confronto dos n coletados com os correspondentes calculados através da equação atrás deduzida, mostra que um desvio de 0,00002 , isto é, de 2 unidades na 5 . $^{\mathrm{a}}$ casa decimal corresponde a um desvio de $1 / 50.000$. Entre os 62 casos confrontados, isto é, $50 \%$ dos casos, apresentam desvios inferiores a 2 unidades na $5 .^{a}$ casa decimal, portanto, com valores inferiores a $1 / 50.000$.

Em 19 casos, cerca de $30 \%$ dos casos, os desvios são iguais ou inferiores a 1/100.000.

Um desvio de 3 unidades na $5 .^{a}$ casa representa $1 / 33333$, com apenas 5 casos constatados.

$\mathrm{O}$ objetivo final deste trabalho foi o de verificar a possibilidade de simplificação de certas operações de campo, durante a rotina de trabalho em poligonação a telurômetro para fins (artográficos ou outros, onde a precisão de medidas de distâncias não fosse além de $1 / 50.000$. Mais precisamente, a idéia é a de eliminar as medidas de temperatura úmida e seca e da pressão barométrica.

\begin{tabular}{l|c|c}
\hline n coletado & n calculado & desvio \\
\hline 1,0003153 & 1,0003440 & $+0,0000287$ \\
1,0002946 & 1,0003490 & $+0,0000544$ \\
1,0003626 & 1,0003405 & $-0,0000221$ \\
1,0003560 & 1,0003256 & $-0,0000304$ \\
1,0003494 & 1,0003606 & $+0,0000112$ \\
1,0003408 & 1,0003690 & $+0,0000282$ \\
1,0003186 & 1,0003461 & $+0,0000275$ \\
1,0003200 & 1,0003455 & $+0,0000255$ \\
1,0003840 & 1,0003576 & $-0,0000268$ \\
1,0003586 & 1,0003679 & $+0,0000093$ \\
1,0003483 & 1,0003590 & $+0,0000107$ \\
1,0003475 & 1,0003546 & $+0,0000071$ \\
1,0003546 & 1,0003367 & $-0,0000179$ \\
1,0003569 & 1,0003358 & $-0,0000211$ \\
1,0003575 & 1,0003425 & $-0,0000150$ \\
1,0003440 & 1,0003570 & $+0,0000130$ \\
1,0003473 & 1,0003356 & $-0,0000117$ \\
1,0003566 & 1,0003811 & $+0,0000245$ \\
1,0003378 & 1,0003425 & $+0,0000047$ \\
1,0003505 & 1,0003405 & $-0,0000100$ \\
1,0003528 & 1,0003406 & $-0,0000122$ \\
1,0003661 & 1,0003347 & $-0,0000314$ \\
1,0003206 & 1,0003469 & $+0,0000263$ \\
1,0003203 & 1,0003427 & $+0,0000224$ \\
1,0003304 & 1,0003058 & $-0,0000246$ \\
1,0003250 & 1,0003549 & $+0,0000299$ \\
1,0003356 & 1,0003293 & $-0,0000063$
\end{tabular}

Entre os índices n coletados, muitos deles foram obtidos através de nomograma da fórmula de Essen-Froome, com um erro gráfico de pelo menos 1 unidade na $5 .^{a}$ casa decimal.

A conclusão a que se pode chegar com base na análise realizada é de que com dados mais refinados, isto é, com a melhora dos dados de observação de campo se possa reduzir os desvios constatados, assegurando assim maior validade para os resultados.

Mesmo com os resultados apresentados parece-nos razoável admitir que, em trabalhos cuja precisão não chegue a $1 / 50.000$, se possa adotar a equação aqui deduzida, eliminando-se as determinações de temperatura e da pressão barométrica, uma vez que se proceda a um cálculo e anotação do coeficiente $\mathrm{Ccr}$, de acordo com a fórmula já indicada, no campo, não somente para o controle de medidas de distâncias zenitais como para a obtenção do índice $n$ de refração, necessário à correção das medidas obtidas pelo telurômetro.

Com o fim de testar a aplicação da fórmula de regressão deduzida neste trabalho, recalculamos, para fins de comparação, uma 


\section{PROGRAMA UTILIZADO PARA O CÁLCULO DAS POLIGONAIS}

0 :

SPC 2 -

1 :

FXD 8; ENT “AZ=?"

,R1, "N=?", R2, "D=?"

,R3, “DIRP=?”, R

4, “AZP=?”, R5, "K1

$=$ ?”, R6 -

2 :

ENT "K2=?", R7, "K

$3=2$ ", R8

3 :

ENT "DIR.GR = ?", R

9, "MIN=?", R10, "S

$\mathrm{EG}=$ ?", R11 -

4 :

$\mathrm{R} 9+\mathrm{R} 10 /, 60+\mathrm{R} 11 / 3600$

$\rightarrow \mathrm{R} 12$ -

5 :

ENT “DIST. G=?",

R13; PRT "DIST. G= ,R13 -

6:

$\mathrm{R} 12+\mathrm{R} 1 \rightarrow \mathrm{R} 1$; IF R1 > 360;

$\mathrm{R} 1-360 \rightarrow \mathrm{R} 1$

7 :

COS R1*R13 $\rightarrow$ R14; R

$14+\mathrm{R} 2 \rightarrow \mathrm{R} 2$; PRT "N=",

R2 -

8 :

SIN R1*R13 $\rightarrow$ R15; R

$15+\mathrm{R} 3 \rightarrow \mathrm{R} 3$; PRT "E="

, R3 -

9:

$(\mathrm{R} 3+\mathrm{R} 15 / 2) * \mathrm{R} 6 * \mathrm{R} 1$

$4 \rightarrow$ R16; R6*R14*R15

$|6 \rightarrow \mathrm{R} 17|-$

$10: *$

$(\mathrm{R} 16-\mathrm{R} 17)(-1) / 36$

$00 \rightarrow$ R18;(R16+R17)

$\mid 3600 \rightarrow$ R19 $\mid-$

11 :

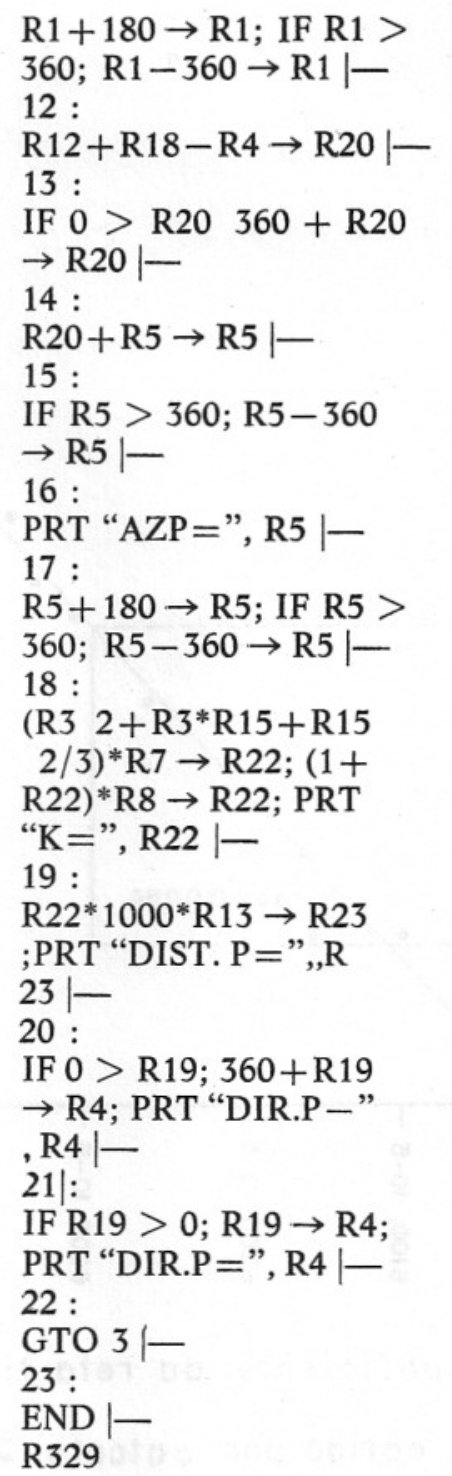

Programado pelo autor deste trabalho 
AJUSTAMENTO GRÁFICO DA RETA DE

CORRELAÇĀO PELOS PONTOS M(Ccri; $\left.n_{i}\right)$

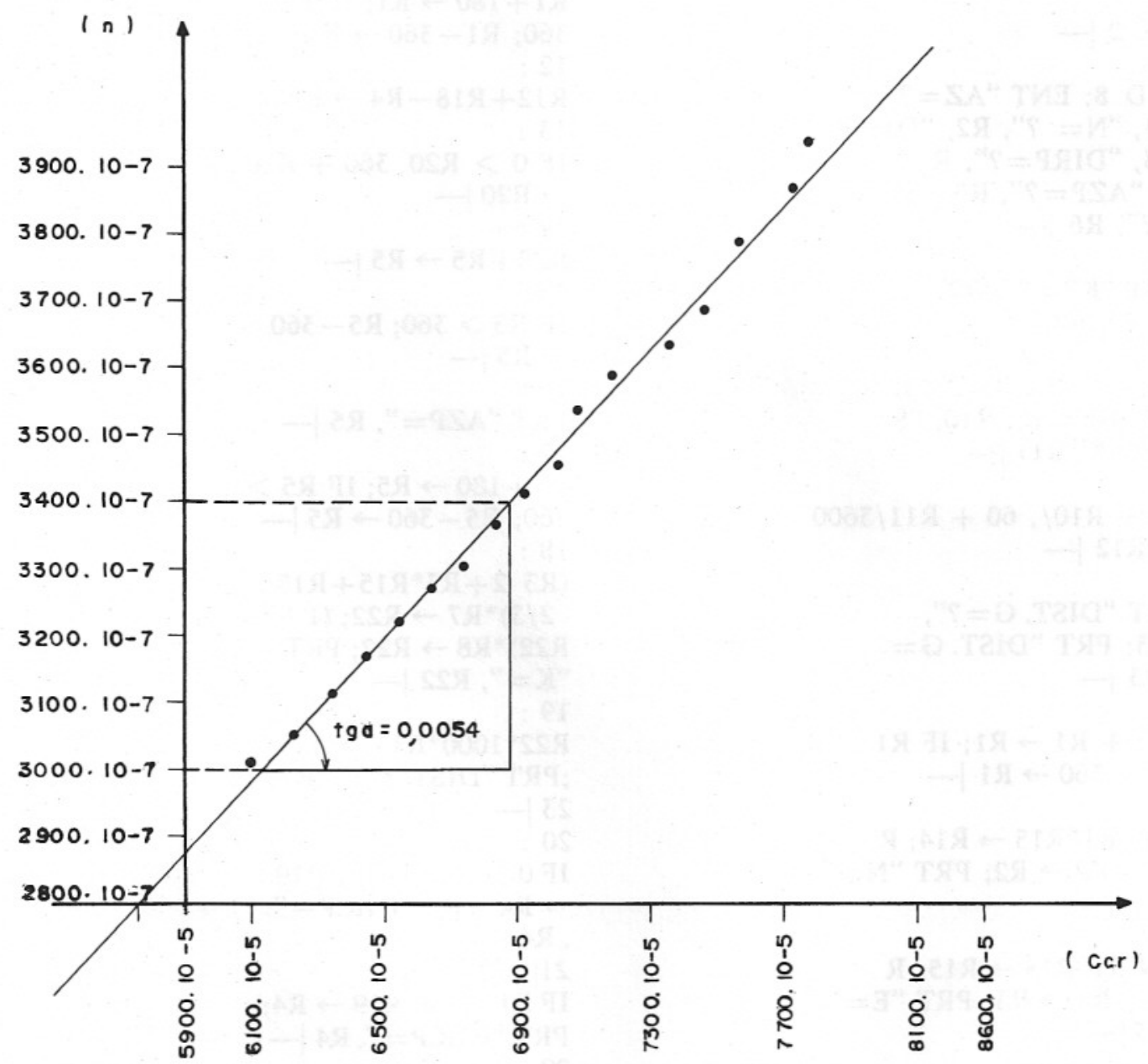

o coeficiente da reta tirada graficamente $e^{\prime} 0,0054$

e o obtido por calculo $\sum X Y$

$$
\overline{\Sigma X 2}=0,0052
$$

da reta de regressão

Figura 2 
poligonal fechada controlada por estações de uma outra poligonal estabelecida para o apoio fotográfico, usado no levantamento cartográfico 1:50.000, do Estado de São Paulo.

A referida poligonal foi desenvolvida para fins de locação de poços tubulares profundos, onde o conhecimento da posição espacial interessava à pesquisa de água subterrânea que o IGG realiza na região da bacia do rio São José dos Dourados.

Sendo o principal interesse a obtenção de maior fidelidade possível nos dados de altimetria, houve grande tolerância na precisão planimétrica, o que em nada altera a comparação que pretendemos fazer.

Na poligonal em questão foi utilizado telurômetro modelo MRA.3, calibrado para obtenção de distância referida a um definido valor de índice de refração, $\mathrm{n}_{0}=1,000325$, de maneira que as distâncias lidas devem ser multiplicadas pelo fator $n_{0} / n$, sendo, pois, introduzida uma modificação na fórmula (4) deduzida:

$$
\begin{aligned}
& \mathrm{n}=0,0052 \mathrm{Crc}+0,9999722 \text { para } \\
& \mathrm{n}^{\prime}=\frac{\mathrm{n}_{\mathrm{o}}}{\mathrm{n}}=\frac{\mathrm{n}_{\mathrm{o}}}{0,0052 \mathrm{Crc}+0,9999722}
\end{aligned}
$$

A poligonal ensaiada consta de 8 lados, com coeficientes $\mathrm{Crc}$ variando de 0,066 a 0,085 , com média 0,074 , isto é, acima do valor médio geral calculado de 0,070 , apresentando condições desfavoráveis de aplicação da fórmula de regressão. Para o valor 0,070 , a fórmula dá um valor de $\mathrm{n}=$ 1,0003362 que difere de $n_{0}$ de 0,000011 .

O cálculo da poligonal foi efetuado numa calculadora eletrônica Hewllet Packard, mod. 9820.A, opção 001, utilizando um programa que aproveitou uma planilha de cálculo idealizada pelo eng. ${ }^{\circ}$ José de Oliveira Quintão para transformação de elementos geodésicos em elementos planos do sistema UTM, muito prática para programação pelo encadeamento dos cálculos, o que permitiu notável simplificação.

Cálculo da coordenada UTM com distâncias

\begin{tabular}{|c|c|c|c|}
\hline Est. azimute plano & $\mathrm{N}$ & $\mathrm{E}$ & dist. plana \\
\hline 1 & $7.710 .184,65$ & $645.711,28$ & \\
\hline $283^{\circ} 46^{\prime} 43,79^{\prime \prime}$ & & & 9245,20 \\
\hline 2 & $7.712 .386,62$ & $636.732,14$ & \\
\hline $188^{\circ} 00^{\prime} 34,58^{\prime \prime}$ & & & 10378,00 \\
\hline 3 & $7.702 .109,86$ & $635.286,07$ & \\
\hline $135^{\circ} 38^{\prime} 25,65^{\prime \prime}$ & & & 6636,17 \\
\hline 4 & $7.697 .365,22$ & $639.925,81$ & \\
\hline $120^{\circ} 26^{\prime} 54,87^{\prime \prime}$ & & & 5814,16 \\
\hline 5 & $7.694 .418,81$ & $644.938,11$ & \\
\hline $62^{\circ} 09^{\prime} 19,35^{\prime \prime}$ & & & 9690,26 \\
\hline 6 & $7.698 .944,89$ & $653.506,40$ & \\
\hline $334^{\circ} 05^{\prime} 16,12^{\prime \prime}$ & & & 7174,93 \\
\hline 7 & $7.705 .398,49$ & $650.371,01$ & \\
\hline $315^{\circ} 46^{\prime} 05,77^{\prime \prime}$ & & & 6680,14 \\
\hline 8 & $7.710 .184,97$ & $645.711,20$ & \\
\hline $321^{\circ} 34^{\prime} 24,69^{\prime \prime}$ & & & 15767,71 \\
\hline 9 & $7.722 .537,50$ & $635.911,41$ & \\
\hline $\begin{array}{l}\text { Coord. de controle } \\
\text { da estação } 9\end{array}$ & $7.722 .531,25$ & $635.910,40$ & \\
\hline Erro de fechamento & $+6,25$ & $+1,01$ & - \\
\hline
\end{tabular}
obtidas pelo processo habitual 


\begin{tabular}{|c|c|c|c|}
\hline \multicolumn{4}{|c|}{$\begin{array}{l}\text { Cálculo com distâncias geodésicas corrigidas } \\
\text { pela fórmula de regressão }\end{array}$} \\
\hline 1 & $7.710 .184,65$ & $645.711,28$ & \\
\hline $283^{\circ} 46^{\prime} 43,79^{\prime \prime}$ & & & 9246,91 \\
\hline 2 & $7.712 .386,62$ & $636.732,14$ & \\
\hline $188^{\circ} 00^{\prime} 34,58^{\prime \prime}$ & & & 10379,92 \\
\hline 3 & $7.702 .109,86$ & $635.286,07$ & \\
\hline $135^{\circ} 38^{\prime} 25,65^{\prime \prime}$ & & & 6637,39 \\
\hline 4 & $7.697 .365,22$ & $639.925,81$ & \\
\hline $120^{\circ} 26 ’ 54,87^{\prime \prime}$ & & & 5815,00 \\
\hline $5 \quad 62^{\circ} 09^{\prime} 19,35^{\prime \prime}$ & $7.694 .418,81$ & $644.938,11$ & 9692,31 \\
\hline - & $7.698 .944,89$ & $653.506,40$ & \\
\hline $334^{\circ} 05^{\prime} 16,12^{\prime \prime}$ & & & 7176,10 \\
\hline $715^{\circ} 46^{\prime} 05,77^{\prime \prime}$ & $7.705 .398,49$ & $650.371,01$ & 6681,21 \\
\hline 8 & $7.710 .184,26$ & $645.711,89$ & \\
\hline $321^{\circ} 34^{\prime} 24,69^{\prime \prime}$ & & & 15769,58 \\
\hline 9 & $7.722 .536,78$ & $635.912,11$ & \\
\hline $\begin{array}{l}\text { Coordenadas de con- } \\
\text { trole da estação } 9\end{array}$ & $7.722 .531,25$ & $635.910,40$ & \\
\hline Êrro de fechamento & $+5,53$ & $+1,71$ & \\
\hline
\end{tabular}

Observe-se que os valores de azimutes permaneceram constantes num e noutro caso, como era lícito esperar, como uma conseqüência do próprio método de cálculo.

No método de transformação de elementos geodésicos em planos, uma pequena alteração de comprimento de lados pouco influencia os resultados, com tendência a diminuir as diferenças observadas nos valores geodésicos, o que é evidente.

\begin{tabular}{rrr}
\hline $\begin{array}{c}\text { Dist. } \\
\text { geodésicas } \\
\text { pelo } \\
\text { processo } \\
\text { normal }\end{array}$ & $\begin{array}{c}\text { Dist. } \\
\text { geodésicas } \\
\text { com } \\
\text { correção } \\
\text { pela } \\
\text { fórmula }\end{array}$ & $\begin{array}{c}\text { Coeficientes } \\
\text { de refração } \\
\text { e curvatura } \\
\text { observadas }\end{array}$ \\
\hline 9246,91 & 9246,56 & 0,075 \\
10379,92 & 10379,92 & 0,068 \\
6637,39 & 6637,19 & 0,076 \\
5815,00 & 5814,54 & 0,085 \\
9692,31 & 9692,04 & 0,075 \\
7176,10 & 7176,66 & 0,066 \\
6681,21 & 6680,89 & 0,068 \\
15769,58 & 15769,56 & 0,074 \\
$\Sigma 71398,42$ & $\Sigma 71397,36$ &
\end{tabular}

No processo usual de cálculo o comprimento total da poligonal geodésica é de $71.398,42$, contra $71.397,36 \mathrm{~km}$ soma de lados planos, acusando uma diferença de $11,85 \mathrm{~m}$.

Com a aplicação da fórmula essa diferença baixou para $9,84 \mathrm{~m}$.

Os erros de fechamento, em coordenadas, referidos às coordenadas de controle da estação 9 são:

processo habitual $+1,01+6,25$

c/ uso da fórmula $+1,71+5,53$

diferenças

$$
0,70 \mathrm{~m} \quad 0,72 \mathrm{~m}
$$

Do exame dos resultados, muitas conseqüências de ordem prática podem ser tiradas.

Por exemplo, quando das transformações de elementos geodésicos (distância e azimute) para elementos planos do sistema UTM, para finalidades cartográficas em escalas de 1:50.000, ou pouco maiores, pouco significado apresenta a tentativa de aumentar a precisão nas medidas obtidas por telurômetro (ou equipamento similar), com várias repetições de medidas finas, o mesmo acon- 
tecendo com medidas angulares, quer horizontais ou verticais.

Isto é evidente pela simples razão de que os cálculos para a citada transformação partem de valores aproximados, tais que qualquer tentativa para a melhora de precisão tem pouco significado (às vezes nenhum), quando comparado com a precisão requerida.

A mesma consideração pode ser feita relativamente às correções envolvendo o índice de refração o qual, além de sujeito a uma variabilidade imprevisível de natureza aleatória, toma valores internos a um definido segmento, conforme verificado anteriormente, com influência nos resultados, compatíveis com a precisão necessária para determinada faixa em torno de 1:50.000.

$\mathrm{O}$ que não nos parece razoável é insistir na obtenção de precisões de medidas, que serão alteradas dentro do aparato de formulações matemáticas, baseado em hipóteses aproximativas, impossíveis de assumirem uma forma analítica exata.

\section{CONCLUSÕES}

As análises objeto deste trabalho resultantes da análise estatística de uma amostragem bastante significativa, levam a conclusões perfeitamente aceitáveis.
As distribuições consideradas são do tipo êrro, portanto sujeitas a leis normais.

a) A fórmula de regressão 4 obtida pode ser aplicada com vantagem em medições com equipamento eletrônico nas condições antes especificadas, pela simplificação operacional que introduz.

b) $\mathrm{Na}$ fórmula 1, como critério de aceitação ou rejeição das observações, devese adotar o valor de referência 0,070 ao invés de 0,067 , como valor mais confiável.

c) Os levantamentos geodésicos para fins cartográficos, nas escalas já analisadas, bem como as poligonais de apoio para amarração em projetos podem adotar com segurança os resultados obtidos.

d) Pontos isolados amarrados a uma poligonal de base, podem ser determinados com precisão altimétrica satisfatória, desde que se localizem a distâncias não superiores a $15 \mathrm{~km}$. No caso de amarração de uma bateria de poços tubulares, a poligonal pode ser planejada para atender ao máximo a recomendação acima.

e) Uma utilização da equação de regressão determinada, seria a sua inclusão fácil na estrutura do programa de computação incluido neste trabalho, que exigiria a introdução de apenas um dado variável, Ccr.

\section{REFERENCIAS BIBLIOGRĀFICAS}

AMERICAN SOCIETY OF PHOTOGRAMMETRY - 1966 - Manual of photogrammetry. 3rd. ed. Falls Church, Va. 2v.

O autor consultou, para realização deste trabalho, os Arquivos da então Divisão de Geografia do Instituto Geográfico e Geológico a partir de 1960. Seção de Levantamentos Básicos.
GMURMAN, V.E. - 1974 - Teoria de las probilidades y estadística matemática. Moscou, Editorial MIR. 388p.

LEVALLOIS, J.J. - 1969-1970 - Géodesie générale. Paris, Eyrolles. 2v. 